01,11

\title{
Возможные механизмы формирования бейнитных колоний
}

\author{
(C) И.К. Разумов \\ Институт фризики металлов УрО РАН, \\ Екатеринбург, Россия \\ E-mail: rik@imp.uran.ru
}

\section{(Поступила в Редакцию 8 августа 2018 г.)}

Обсуждаются возможные механизмы бейнитного превращения в сталях. В соответствии с известными моделями роста видманштеттова феррита, иглообразная форма бейнитных планок определяется анизотропией поверхностной энергии. Однако механизмы размножения планок в верхнем и нижнем бейните предположительно различаются. Верхний бейнит является диффузионно-контролируемым превращением, при котором возможен автокатализ перлитного типа, обусловленный образованием цементита на границе феррита. Нижний бейнит формируется при более низкой температуре бездиффузионным путем, причем ветвление выделений либо автокатализ планок могут быть обеспечены за счет понижения энергии системы при размещении структурных дефектов на интерфейсе выделений, так что существование характерного размера планки энергетически обусловлено (эффект Вейсмюллера). Совместное действие различных механизмов автокатализа приводит к многообразию возможных бейнитных форм.

Работа выполнена в рамках государственного задания по темам „Магнит“ № АAАА-А18-118020290129-5 и „Структура“ № AААА-А18-118020190116-6.

DOI: 10.21883/FTT.2019.02.47116.212

\section{1. Введение}

Бейнит (наряду с ферритом, перлитом и мартенситом) является одним из возможных продуктов $\gamma-\alpha$ превращения при охлаждении стали [1]. Легированные бейнитные стали высокой и средней прочности нашли широкое применение в крупногабаритных сварных конструкциях, автомобильной индустрии, энергетике. Однако несмотря на обширные экспериментальные и теоретические исследования, механизмы формирования бейнитной микроструктуры остаются не вполне ясными [2].

Верхний бейнит образован планками видманштеттова феррита $(\alpha)$, разделенными прослойками цементита $(\theta)$. В отличие от перлита когерентность решетки на интерфейсе $\gamma / \alpha$ частично сохраняется, а выделения $\alpha$ и $\theta$ могут расти некооперативно (в верхнем бейните сначала выделяется феррит [1,3]), хотя соотношение объемных долей $\alpha$ и $\theta$ на развитых стадиях роста колонии близко к константе [4]. Нижний бейнит формируется при более низкой температуре и состоит из ферритных планок, разделенных прослойками аустенита; цементит выпадает на поздних стадиях превращения, как на границах, так и в объеме ферритных планок.

В настоящее время установлено, что бейнитное превращение является сдвиговым по природе [2], но продолжается спор о том, лимитируется ли его скорость диффузией углерода или релаксацией напряжений $[1,5,6]$. Согласно Бхадешиа [1], оба вида бейнита реализуются ниже температуры $T_{0^{\prime}}$ (см. рис. 1 , области $\mathrm{UB}_{1}, \mathrm{LB}$ ), то есть в области фазовой диаграммы, где превращение $\gamma \rightarrow \alpha$ возможно в отсутствие диффузии углерода. Более того, обнаружено несколько режимов $\gamma-\alpha$ превращения в чистом железе, в зависимости от температуры [7], которые могут ассоциироваться с двумя бейнитными, и/либо с двумя мартенситными превращениями (изотермическим и атермическим) [8].

Существуют, однако, экспериментальные факты [3], согласно которым структура, морфологически эквивалентная верхнему бейниту, возникает в интервале тем-

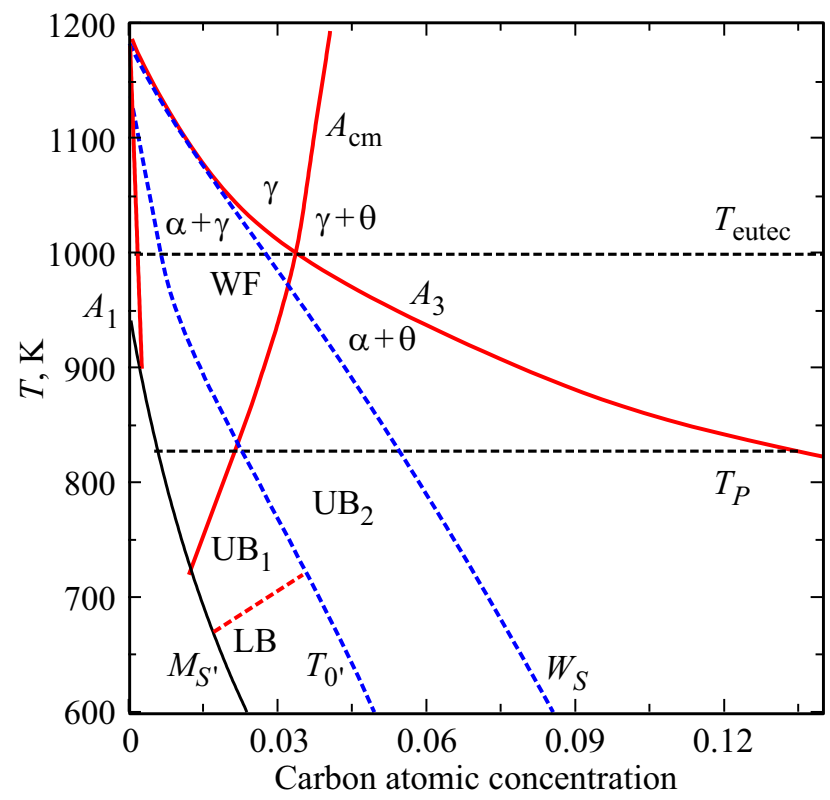

Рис. 1. Диаграмма превращений в углеродистой стали. $A_{1}, A_{3}, A_{c m}$ - границы двухфазных областей $\gamma+\alpha, \gamma+\theta$; $W_{S}, M_{S}$ - стартовые линии видманштеттова феррита [5] и мартенсита, $T_{0^{\prime}}$ - линия равенства свободных энергий фаз $\gamma$ и $\alpha$ (с учетом вклада напряжений) при неизменном содержании углерода $[1] ; T_{P}$ - температура старта перлитного автокатализа [10]; WF, $\mathrm{UB}_{1}, \mathrm{UB}_{2}, \mathrm{LB}$ - области видманштеттова феррита, верхнего и нижнего бейнита. 


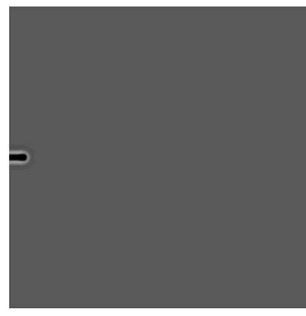

$t=0$

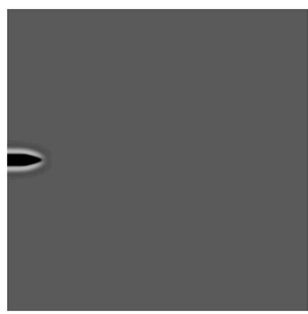

$t=0.01$

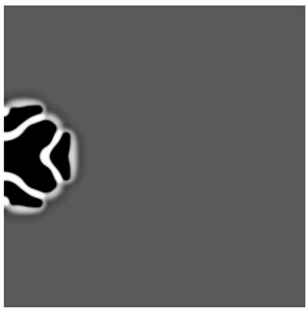

0.13

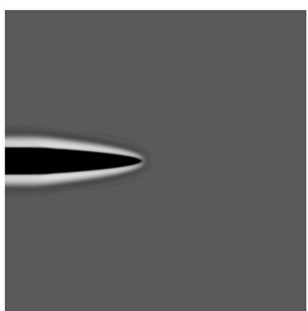

0.05

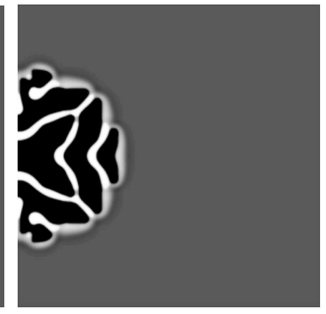

0.21

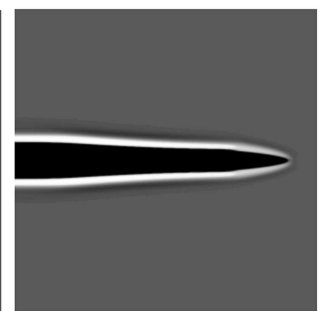

0.11

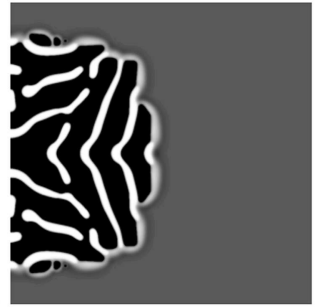

0.32

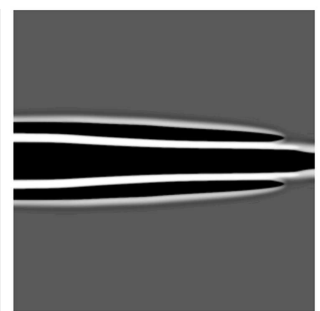

0.14

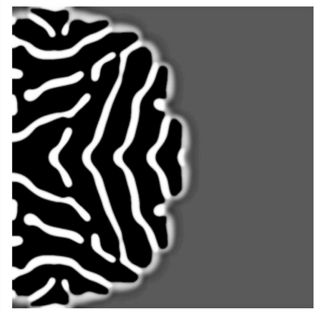

0.38

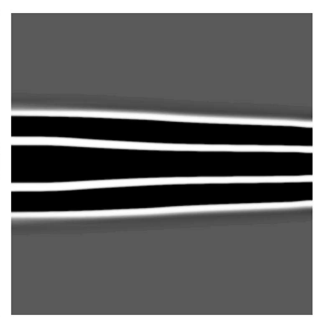

0.19

Рис. 2. Автокаталитический распад аустенита (серый цвет) на феррит (черный) и цементит (белый) в рамках модели [10] с изотропной $\left(\sigma_{x} / \sigma_{y}=1\right.$, верхний ряд, перлитная колония) и анизотропной $\left(\sigma_{x} / \sigma_{y}=5\right.$, нижний ряд, аналогия верхнего бейнита) поверхностной энергией; $T=625 \mathrm{~K}$, концентрация углерода $c_{0}=0.06$.

ператур $T_{0}<T<W_{s}\left(W_{s}-\right.$ стартовая линия видманштеттова феррита, выше которой превращение $\gamma-\alpha$ не может реализоваться путем кооперативного смещения атомов железа [5]), см. рис. 1, область $\mathrm{UB}_{2}$. В этом случае формирование верхнего бейнита контролируется диффузией углерода, причем области верхнего бейнита и перлита на фазовой диаграмме перекрываются [3,9]. Для перлита характерна полная потеря когерентности решетки на границе $\gamma / \alpha$, в то время как для бейнитного феррита она частично сохраняется. Запуск превращения по тому или иному сценарию зависит, возможно, от стартовых условий зарождения первого выделения феррита.

Другим неясным вопросом является механизм размножения ферритных планок в бейнитной колонии. Предложенная в работах [10-13] модель структурнофазовых превращений в стали с элементами первопринципной параметризации позволила построить основные линии фазовой диаграммы в системе „железо-углерод“ в согласии с экспериментом и провести моделирование ферритного, перлитного и мартенситного превращений. Однако она не позволила наблюдать рост бейнитной колонии. Если размножение планок реализуется по механизму перлитного автокатализа, как это предполагалось в работах $[14,15]$, тогда в рамках этой модели утрачивается критерий различия перлита и бейнита. К тому же хорошо установлено, что по крайней мере нижний бейнит может формироваться в отсутствие цементита. С другой стороны, упругие напряжения стимулируют автокатализ ферритных пластин по мартенситному типу (двойникованием), однако в экспериментах ферритные планки в бейнитной колонии имеют одинаковую кристаллографическую ориентировку. По мнению Бхадешиа: „It seems that strain-induced autocatalysis does not play an important role in bainite formation" [1].

\section{2. Результаты моделирования}

Иглообразную форму выделений видманштеттова феррита обычно объясняют анизотропией поверхностной энергии [16,17], которая для простоты не учитывалась в $[11,12]$. Вероятно, эта анизотропия теряется при нарушении когерентности решетки на границе $\gamma / \alpha$, приводя к смене сценариев „верхний бейнит/перлит“. На рис. 2 представлено моделирование роста колонии выделений феррита и цементита в рамках модели автокаталитического распада [10], в отсутствие (верхний ряд) и при наличии (нижний ряд) анизотропии поверхностной энергии, которая учтена заменой $\sigma(\nabla c)^{2} \rightarrow \sigma_{x}\left(\nabla_{x} c\right)^{2}+\sigma_{y}\left(\nabla_{y} c\right)^{2}$ в функционале свободной энергии Гинзбурга-Ландау. Можно видеть, что кинетика превращения различается в этих случаях. В первом случае имеет место кооперативный рост выделений $\alpha$ и $\theta$. Во втором случае сначала возникает игла феррита, которая уширяется со временем, а цементит появляется на следующем этапе. Данный механизм относится прежде всего к верхнему бейниту в области $\mathrm{UB}_{2}$ (см. рис. 1). Бейнит в области $\mathrm{UB}_{1}$ может формироваться по этому же сценарию: при достаточно высокой температуре углерод, вытесняясь из феррита, образует „запирающую“ оболочку, после чего превращение становится диффузионно-контролируемым. Однако этот механизм не объясняет рост колоний нижнего бейнита в отсутствие цементита. По-видимому, существует дополнительный механизм размножения ферритных планок, не связанный ни с цементитом, ни с напряжениями.

Эксперименты показывают существование характерного размера бейнитных планок (как по длине, так и по ширине). Это не может объясняться упругим равновесием с матрицей, так как релаксация напряжений при длительной выдержке не приводит к изменению 

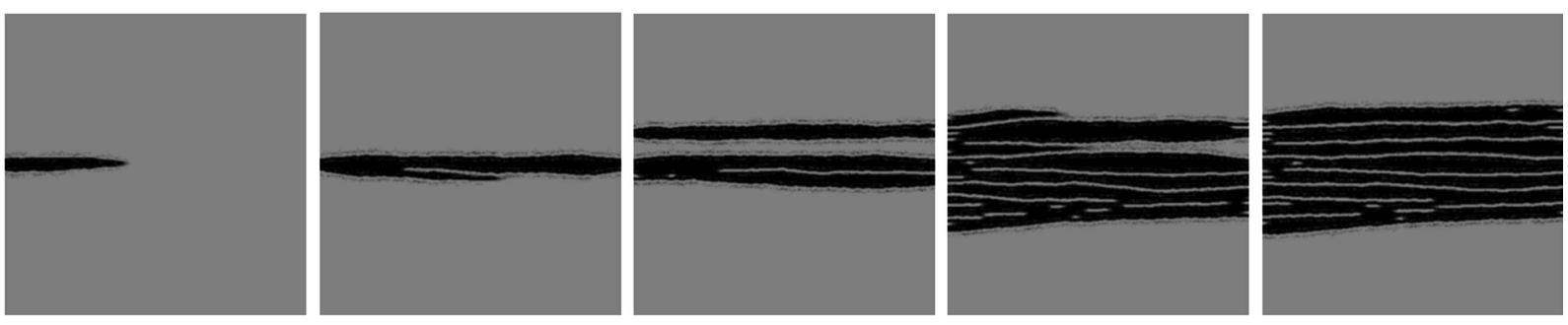

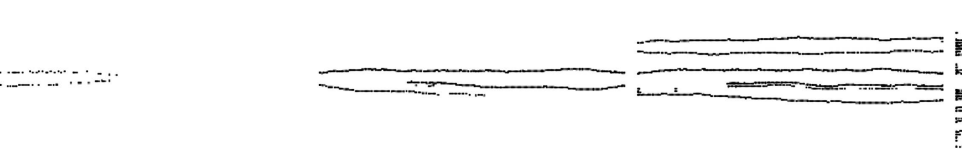

$N=2 \cdot 10^{7}$

$4 \cdot 10^{8}$

$10^{9}$

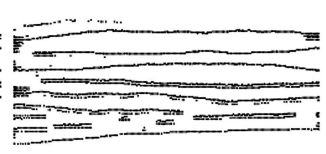

$2 \cdot 10^{9}$

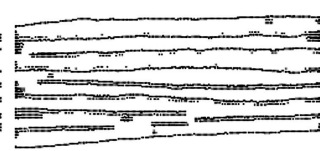

$2.4 \cdot 10^{9}$

Рис. 3. Рост колонии подобной нижнему бейниту, на квадратной сетке $400 \times 400$ в условиях генерации и скопления дефектов на интерфейсе выделений (верхний ряд) и соответствующее распределение дефектов (нижний ряд). $g_{\alpha}=0, g_{\gamma}=4, g_{b} / k T=58$, $\sigma_{\phi \xi} / k T=-0.07, \sigma_{\phi}^{x} / k T=10^{-4}, \sigma_{\phi}^{y}=10^{-3}, \sigma_{\xi} / k T=10^{-2}, C_{\xi}=0.03, Q_{0}=2.5 \cdot 10^{-5}$. Время выражено числом итераций $N$.

размера планок. К тому же, согласно Бхадешиа [1], планки не являются изолированными, а соединены перемычками, которые не всегда видны в двумерном сечении образца. В последнем случае речь идет, скорее, о сильном ветвлении единственного выделения феррита, a не об автокаталитическом размножении планок. Отсюда можно заключить, что бейнитная колония подобна системе с нулевой поверхностной энергией, то есть ограничение роста и слияния выделений феррита обусловлено энергетической выгодностью границ раздела $\gamma / \alpha$. Подобный эффект рассматривался Вейсмюллером для рекристаллизации зерен в условиях зернограничной сегрегации (ЗГС) примеси [18] и был экспериментально подтвержден [19]. Было показано, что в некоторых системах благодаря ЗГС, вопреки собирательной рекристаллизации, достигается равновесный размер зерен, при котором энергия их границ является нулевой. В случае бейнитного превращения роль ЗГС могут играть скопления дислокаций, которые генерируются в результате пластической деформации при росте планки и имеют наименьшую энергию при размещении на интерфейсе $\gamma / \alpha$, компенсируя несоответствие решеток. В таком случае существование протяженных интерфейсов, при отсутствии заметной тенденции к слиянию выделений феррита, становится энергетически выгодным.

Для качественной демонстрации эффекта сформулируем простую модель без углерода, в которой на интерфейсе $\gamma / \alpha$ генерируются не дислокации, а точечные дефекты, имеющие отрицательную энергию связи с интерфейсом. Функционал свободной энергии Гинзбурга-Ландау (Г.-Л.) запишем в виде:

$$
\begin{aligned}
F=\int\left(f_{b}(\phi)\right. & +\sigma_{\phi}^{x}\left(\nabla_{x} \phi\right)^{2}+\sigma_{\phi}^{y}\left(\nabla_{y} \phi\right)^{2} \\
& \left.+\sigma_{\phi \xi} \xi(\nabla \phi)^{2}+A \xi+\sigma_{\xi}(\nabla \xi)^{2}\right) d r .
\end{aligned}
$$

Здесь $\phi-$ неконсервативный параметр порядка, характеризующий превращение $(\phi=0$ в аустените, $\phi= \pm 1$ в феррите), $\xi$ - плотность дефектов, $f_{b}(\phi)-$ плотность энергии вдоль бейновского пути, $\sigma_{\phi}^{x(y)}-$ параметры анизотропной поверхностной энергии, $\sigma_{\phi \xi}-$ энергия взаимодействия дефектов с интерфейсом выделений, $A \xi-$ собственная энергия дефектов, $\sigma_{\xi}(\nabla \xi)^{2}-$ вспомогательный вклад, препятствующий кластеризации дефектов. Бейновский путь определим полиномом 6 степени аналогично [12]

$$
\begin{aligned}
f_{b}(\phi)= & 2\left(g_{\alpha}-g_{\gamma}+g_{b} / 6\right)\left(\phi^{2}-\phi^{4} / 2\right) \\
& +g_{b}\left(\phi^{6} / 3-\phi^{4} / 2\right)+g_{\gamma},
\end{aligned}
$$

где $g_{\alpha(\gamma)}$ - плотность энергии в феррите (аустените), $g_{b}$ характеризует высоту барьера между этими состояниями. Заметим, что хотя собственная энергия дефектов $A \xi$ может быть большой, она не зависит от конфигурации и не сказывается на фазовом составе; поэтому полагаем далее $A=0$. Учитывая качественный характер обсуждения, параметры модели выбираются достаточно произвольно.

Для анализа эволюции системы используем метод Монте-Карло на квадратной сетке $400 \times 400$, с зеркально-симметричными граничными условиями. В каждом узле сетки (который соответствует физически малому объему материала) мгновенные точечные значения $\bar{\phi}_{i}, \bar{\xi}_{i}$ принимают значения 0 либо 1. Среднеполевые параметры порядка $\phi, \xi$, присутствующие в функционале Г.-Л. (1), рассчитываем усреднением $\bar{\phi}_{i}, \bar{\xi}_{i}$ по трем координационным сферам. На каждой итерации один из узлов сетки выбирается случайным образом. Значение $\bar{\phi}_{i}$ в этом узле может измениться на противоположное, а значение $\bar{\xi}_{i}$ - путем обмена с одним из соседних узлов. Смена значений происходит с вероятностью в алгоритме Метрополиса, при расчете которой используется функционал Г.-Л. (1). Кроме того, если выбранный узел свободен от дефекта $\left(\bar{\xi}_{i}=0\right)$, реализуется попытка 
генерации дефекта в этом узле $\left(\bar{\xi}_{i}=1\right)$ с вероятностью

$$
P=-Q_{0}(\delta F / \delta \xi)=Q_{0}\left(\sigma_{\phi \xi}(\nabla \phi)^{2}+2 \sigma_{\xi} \Delta \xi\right) .
$$

Выражение (3) означает, что дефекты генерируются в основном на границе раздела $\gamma / \alpha$. Генерация дефектов прекращается, если в окрестности выделенного узла достигнута их предельная концентрация $C_{\xi}$.

На рис. 3 представлена типичная эволюция при старте из одиночного зародыша феррита. На начальной стадии иглообразная форма планки обусловлена анизотропией поверхностной энергии. Уширение планки тормозится скоплением дефектов на интерфейсе, что в итоге определяет ширину планки. Дальнейшая эволюция происходит ветвлением, либо зарождением новых планок. При этом в участках скопления дефектов, в силу $\sigma_{\phi \xi}<0$, предпочтительным состоянием остается аустенит, а точнее межфазная граница $\gamma / \alpha$. На развитых стадиях формируется колония, качественно подобная нижнему бейниту.

\section{3. Выводы}

Таким образом, проведенный анализ позволяет предположить, что формирование верхнего бейнита является диффузионно-контролируемым превращением, в котором морфология выделений определяется анизотропией поверхностной энергии аналогично видманштеттову ферриту, а размножение планок осуществляется по механизму перлитного автокатализа с участием цементита. В отличие от этого формирование нижнего бейнита является бездиффузионным превращением, в котором размножение/ветвление планок осуществляется за счет эффекта Вейсмюллера, то есть в результате отрицательной энергии связи дефектов, генерируемых в процессе роста выделений, с интерфейсом $\gamma / \alpha$, так что бо́льшая площадь границ раздела $\gamma / \alpha$ оказывается энергетически выгодной.

Автор признателен Ю.Н. Горностыреву и М.И. Кацнельсону за полезные обсуждения сценариев и механизмов структурно-фазовых превращений в сталях.

\section{Список литературы}

[1] H.K.D.H. Bhadeshia. Bainite in steels.IOM Communications Ltd, London (2001).

[2] L.C.D. Fielding. Mater. Sci. Tech. 29, 4, 383 (2013).

[3] О.П. Морозов, В.М. Счастливцев, И.Л. Яковлева. ФММ, 2, 150 (1990).

[4] P. Vasudevan, L.W. Graham, H.J. Axon. JISI 190, 386 (1958).

[5] M. Hillert, L. Hoglund, J. Agren. Met. Mater. Trans. A 35, 3693 (2004).

[6] В.М. Счастливцев. МиТОМ 601, 7, 24 (2005).

[7] Д.А. Мирзаев, М.М. Штейнберг, Т.Н. Пономарева, В.М. Счастливцев. ФММ 47, 1, 125 (1979).

[8] В.А. Лободюк, Э.И. Эстрин. УФН 175, 7, 745 (2005).

[9] В.М. Счастливцев, Д.А. Мирзаев, И.Л. Яковлева. Перлит в углеродистых сталях.УрО РАН, Екатеринбург (2006).
[10] I.K. Razumov, Yu.N. Gornostyrev, M.I. Katsnelson. Phys. Rev. Appl. 7, 014002 (2007).

[11] I.K. Razumov, Yu.N. Gornostyrev, M.I. Katsnelson. J. Phys. Condens. Matter 25, 135401 (2013).

[12] I.K. Razumov, D.V. Boukhvalov, M.V. Petrik, V.N. Urtsev, A.V. Shmakov, M.I. Katsnelson, Yu.N. Gornostyrev. Phys. Rev. B 90, 094101 (2014).

[13] И.К. Разумов. ФТТ 59, 1885 (2017).

[14] A. Hultgren. Trans. ASM 39, 915 (1947).

[15] H.J. Lee, G. Spanos, G.J. Shiflet, H.I. Aaronson. Acta Met. 36, 1, 1129 (1988).

[16] A. Yamanaka, T. Takaki, Y. Tomita. Mater. Trans. 47, 11, 2725 (2006).

[17] A. Bhattacharya, K. Ankit, B. Nestler. Acta Mater. 123, 317 (2016).

[18] J. Weissmüller. Nanostruct. Mater. 3, 1-6, 261 (1993).

[19] M. Saber, C.C. Koch, R. Scattergood. Mater. Res. Lett. 3, 2, 65 (2015).

Редактор Ю.Э. Китаев 